\title{
Efficient, high peak power Tm:YLF laser
}

\author{
Lukasz Gorajek* $^{*}$ \\ Institute of Optoelectronics, Military University of Technology, Kaliskiego 2, 00-908 Warszawa, Poland
}

Received August 22, 2013; accepted September 30, 2013; published September 30, 2013

\begin{abstract}
We demonstrate efficient generation of a diode pumped, passively cooled Tm:YLF laser, end-pumped by a $25-\mathrm{W}$ fiber coupled laser diode. Over $5 \mathrm{~W}$ of output power was achieved for a $25-\mathrm{W}$ pumping laser and for an elongated $120-\mathrm{mm}$ cavity. For the free-running and Q-switching regimes, the output spectrum was centered at 1908-nm with a linewidth less than $7 \mathrm{~nm}$. In the experiments on active Qswitching, up to $5.5 \mathrm{~mJ}$ of output energy was demonstrated with a pulse duration of $11 \mathrm{~ns}$, corresponding to $\sim 0.5 \mathrm{MW}$ of peak power.
\end{abstract}

The mid-infrared spectral range $(\sim 1800-5000 \mathrm{~nm})$ is an area of particular interest in leading laboratories in the world. This interest has been mainly driven by the fact that this spectral band contains many characteristic absorption lines of numerous chemical and biological compounds, which makes it vital for applications in e.g. spectroscopy, chemical fingerprinting, security, remote sensing and many others. Many of these applications usually require the use of suitable laser sources operating at wavelengths beyond $1.8 \mu \mathrm{m}$, like thulium solid-state [12] and fiber [3-5] lasers, holmium lasers [6,7], semiconductor crystals lasers with chromium or iron doping $\left(\mathrm{Cr}: \mathrm{A}_{\mathrm{II}} \mathrm{B}_{\mathrm{VI}}\right)[8]$, parametric generators [8] and midinfrared supercontinuum sources [e.g. 10-12].

The main goal of this research was to develop the laser set-up showing the feasibility of q-switched, diode pumped bulk laser based on $\mathrm{Tm}^{3+}$ :YLF crystal, operating at $\sim 1.9-2 \mu \mathrm{m}$ wavelength. As a result of the cross relaxation process (quantum efficiency $\sim 2$ ), the effective optical efficiency of diode pumped Tm lasers can be comparable to typical efficiencies of diode pumped neodymium lasers. Moreover, owing to a wide absorption bandwidth of thulium doped crystals, the same pumping diodes operating at $\sim 0.8 \mu \mathrm{m}$ wavelength can be used.

In the experiment we used a thulium-doped crystal with a $3.5 \%$ thulium ions concentration and cylindrical geometry with a $3 \mathrm{~mm}$ diameter and $10 \mathrm{~mm}$ length. The crystal was cut along the a-axis. The medium was mounted in a copper heat sink, which was placed on an aluminum radiator. The radiator was cooled by two $3 \mathrm{~W}$ electrical fans providing an air flow of about $67 \mathrm{~m}^{3} / \mathrm{h}$.

Two different fiber coupled bars were used as pump sources. The maximum available output power of the first one (PD1) was about $70 \mathrm{~W}$ at a wavelength of $792 \mathrm{~nm}$. The

\footnotetext{
*E-mail: lgorajek@wat.edu.pl
}

diode was mounted on a copper heat sink which temperature was stabilized by four Peltier's elements (each one $50 \mathrm{~W}$ ). The hot surface of the TEC was connected to the central water cooling system. The output power of the second diode (PD2) was about $25 \mathrm{~W}$. It was cooled in the same way as the previous one. The central wavelength of the pump light changed with the temperature of the diode (which was utilized to match the laser linewidth to the absorption band of Tm:YLF crystal), but also with a current amplitude and duty factor. Due to the significant influence of current parameters influencing the wavelength of pump radiation, the optimal operation was possible only for one working point. The laser diode spectrum and absorption spectrum of one of the crystals are presented in Fig. 1.

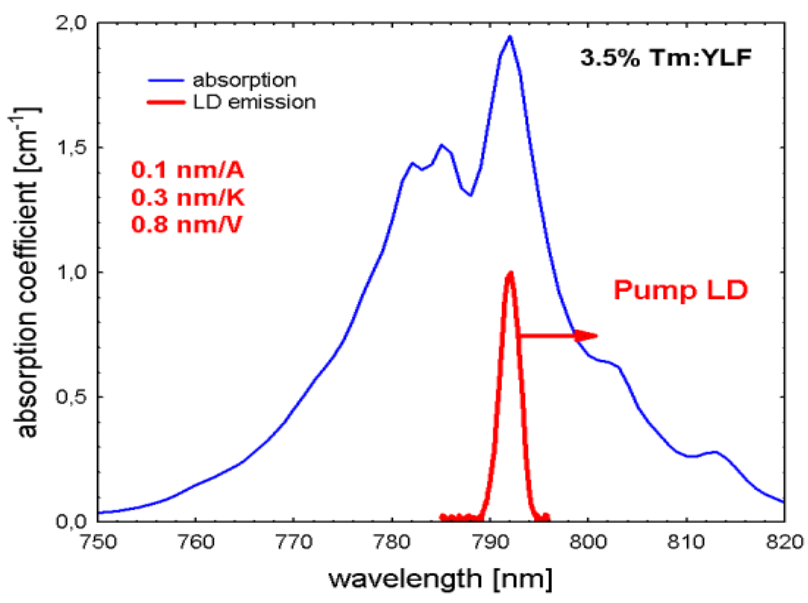

Fig. 1. Pumping diode emission spectrum and active medium absorption spectrum for non polarized pump.

Laser diode marked as PD1 was used to investigate absorption properties of the laser crystal and PD2 was used in generation characterization.

Figure 2 presents the scheme of a laser resonator. The diode radiation coming out of the pigtail fiber $(200 \mu \mathrm{m}$ core, $0.22 \mathrm{NA}$ ) was focused into the active medium by a set of two lenses with anti-reflection coatings $(22 \mathrm{~mm}$ and $33 \mathrm{~mm}$ focal length), throughout a flat dichroic mirror, resulting in approximately an $0.3 \mathrm{~mm}$ pump beam diameter inside the crystal. The output coupler was characterized by a transmission of $30 \%$. To obtain pump and laser 
resonator mode matching, the radius of the mirror curvature was $200 \mathrm{~mm}$. The fundamental resonator mode diameter inside thulium doped crystal was about $280 \mu \mathrm{m}$.

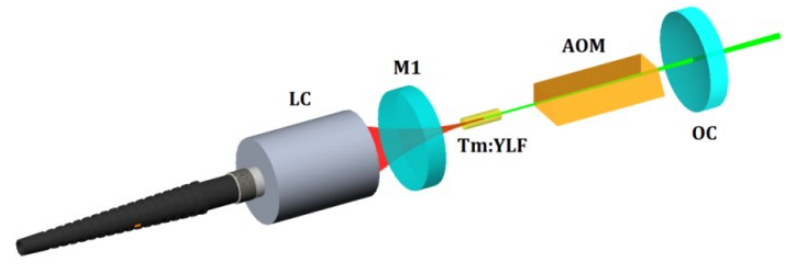

Fig. 2. Scheme of the resonator

The preliminary experiments included absorption spectrum measurements and absorption saturation determination. The absorption properties of investigated samples were determined in the setup shown in Fig. 3. For the estimation of unabsorbed pump power, a dichroic mirror was used. It also prevented recording spontaneous emission affecting the accuracy of the laser output. Absorption efficiency measurements are presented in Fig. 4. The decrease in the absorption with an increase in the pump power is due to the pump beam saturation.

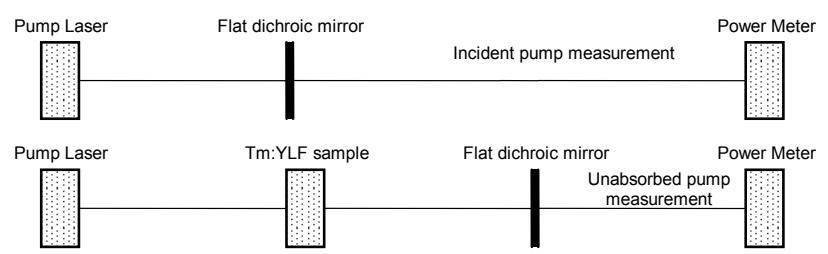

Fig. 3. Experimental setup for the pump absorption measurements.

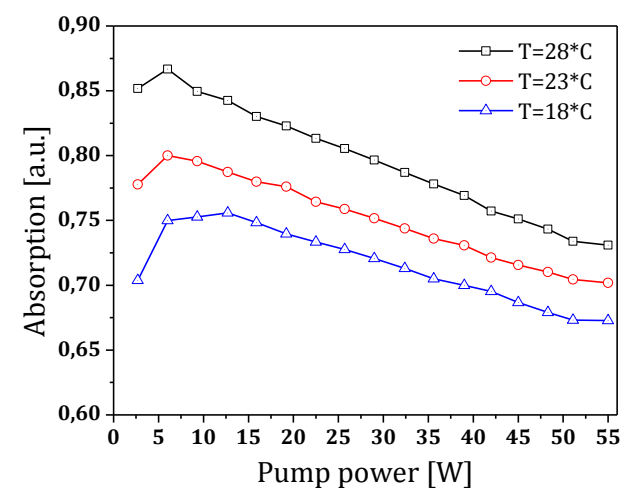

Fig. 4. Absorption efficiency of the Tm:YLF crystals measured for different temperature of pumping laser diode.

In free-running regime examination, Tm:YLF sample was placed inside a linear resonator (Fig. 2). The length was about $120 \mathrm{~mm}$ to provide space for placing an AO-modulator. The pump repetition rate was set to be $20 \mathrm{~Hz}$ and the pulse duration was about 5ms. This configuration, for $10 \%$ duty factor, enabled us to completely diminish laser generation by an AO-modulator when the radio frequency power was on. For the optimized laser, the output power was measured to be over $5 \mathrm{~W}$ by $50 \%$ of slope efficiency. The results of the experiment are presented in Fig. 5.

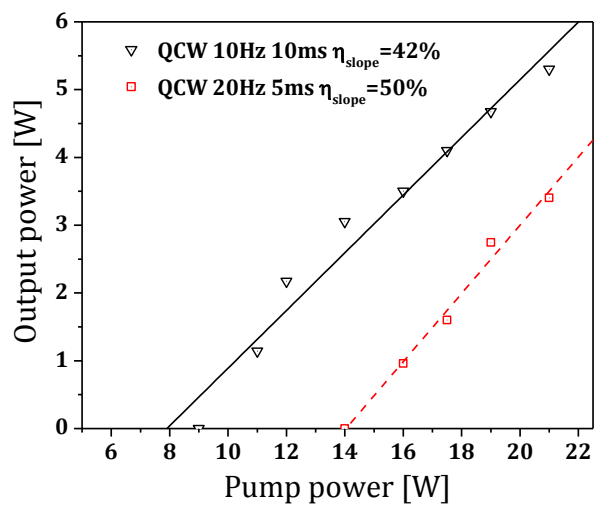

Fig. 5. Output power vs. incident pump power, 10\% pumping duty factor.

An acousto-optic modulator, mounted on a copper heatsink, and operating at a radio frequency of $40.7 \mathrm{MHz}$ with a maximum power of $20 \mathrm{~W}$ was used for Q-switching. For the maximum RF power of acousto-optic modulator, the diffraction angle was about $7 \mathrm{mrad}$. At the maximum output of the laser at $10 \mathrm{~ms}$ pumping pulses, the $\mathrm{AO}$ modulator was unable to hold off generation. Our first step was to determine the maximum available output energy in free-running, for which the acousto-optic modulator can hold off oscillations for switch on state by shortening pumping pulse duration to $5 \mathrm{~ms}$. The laser output was horizontally polarized (perpendicularly to caxis of the Tm:YLF crystal).

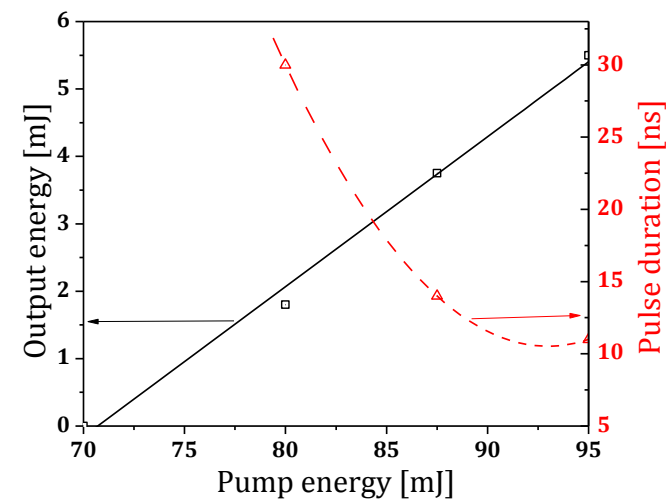

Fig. 6. Output energy and pulse duration vs. incident pump energy.

The results of measurements of pulse duration and energy for a low duty factor of $10 \%$ (20Hz pump repetition rate and $5 \mathrm{~ms}$ pump duration) are shown in 
Fig. 6. The shortest pulses of $11 \mathrm{~ns}$ duration and $5.5 \mathrm{~mJ}$ energy corresponding to $0.5 \mathrm{MW}$ of peak power were demonstrated for the best case of stable output (Fig. 7).

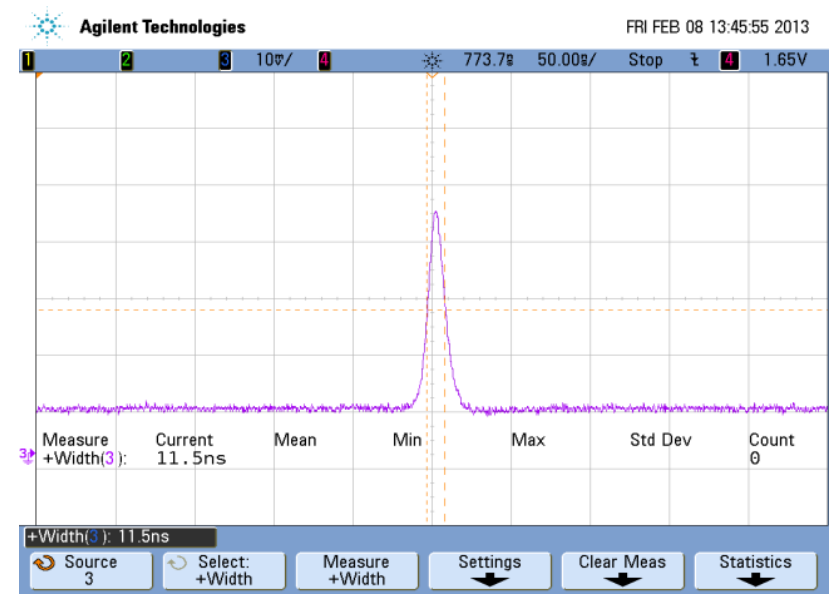

Fig. 7. Half megawatt laser pulse with energy $5.5 \mathrm{~mJ}$.

Beam quality measurements were conducted by means of a Spiricon3 Pyrocam camera. The output laser beam diameter was measured along the propagation axis after focusing lens (300 $\mathrm{mm}$ focal length). The results were approximated with a parabolic propagation law for the Gaussian beam (Fig. 8). Laser beam parameters were calculated to be: $3.5 \mathrm{mrad}$ divergence angle and $\mathrm{M}^{2}$ parameter less than 1.15 .

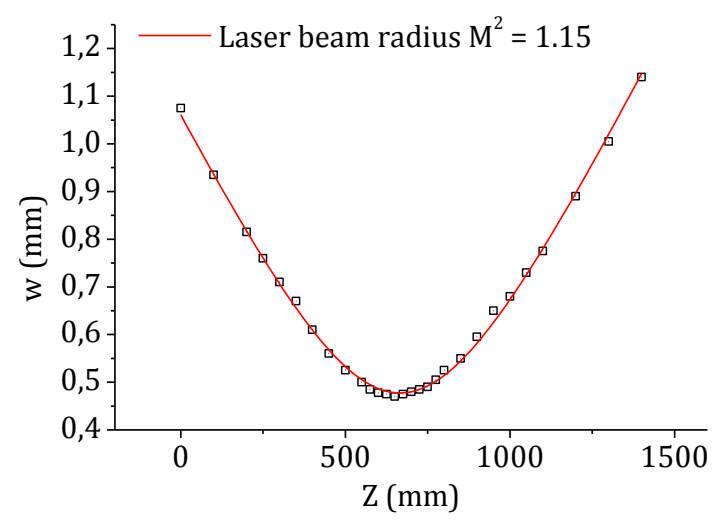

Fig. 8. Output beam radius as a function of the distance from the focusing lens.

We demonstrated efficient generation of a diode pumped Tm:YLF laser, end-pumped by a $25 \mathrm{~W}$ fiber coupled laser diode bar. The incident pump density exceeded the saturation pump density over 5 times. As a result, the drawbacks of quasi-three-level scheme were mitigated. The best output characteristics (slope and maximum power) were obtained for out-coupling losses of $30 \%$ evidencing a high roundtrip gain for maximum pump power. Over $5 \mathrm{~W}$ of output power was demonstrated for a
$25 \mathrm{~W}$ pumping laser for a $120 \mathrm{~mm}$ cavity. For the freerunning and Q-switching regimes, the output spectrum was centered at $1908 \mathrm{~nm}$ with linewidth less than $7 \mathrm{~nm}$. In the experiments on active Q-switching realized with the use of an acousto-optic modulator, up to $5.5 \mathrm{~mJ}$ of output energy was demonstrated. Further pulse energy scaling up was limited by the damage of laser components. For a $20 \mathrm{~Hz}$ repetition rate and a $10 \%$ pump duty cycle, near $0.5 \mathrm{MW}$ of peak power in a $11 \mathrm{~ns}$ duration pulse was achieved. The output laser beam was of very good quality. The divergence angle and $\mathrm{M}^{2}$ factor were measured to be $3.5 \mathrm{mrad}$ and 1.15 , respectively.

The output parameters of the developed lasers, especially relatively high pulse energy in the q-switched regime, make the laser a promising pumping source for e.g. $\mathrm{Cr}^{2+}: \mathrm{ZnSe}$ laser, holmium lasers or ZGP-based optical parametric oscillators operating in the mid-IR spectral region.

This research has been supported by the Polish National Science Center (Project No. NCN2012/05/B/ST7/00088) and Foundation for Polish Science.

\section{References}

[1] J.K. Jabczynski, L. Gorajek, Laser Phys. Lett. 6 (2), 109 (2009).

[2] J.J Sha, D.Y. Shen, T. Zhao, X.F. Yan, Laser Phys. Lett. 10, 075801 (2013).

[3] S. Guillemet, Proc. SPIE 8237, 82373V (2012).

[4] J. Swiderski, M. Maciejewska, J. Kwiatkowski, M. Mamajek, Laser Phys. Lett. 10, 015107 (2013).

[5] J. Swiderski, M. Michalska, Opt. Lett. 38, 1624 (2013).

[6] J. Kwiatkowski, J.K. Jabczynski, L. Gorajek, W. Zendzian, H. Jelinkowa, J. Sulc, M. Nemec, P. Koranda, Laser Phys. Lett. 6(7), 531 (2009).

[7] J. Kwiatkowski, J.K. Jabczynski, W. Zendzian, J. Swiderski, L. Gorajek, L. Galecki, Laser Phys. Lett. 8(4), 281 (2011).

[8] A. Gallian, V.V. Fedorov, Opt. Expr. 14, 11694 (2006).

[9] D. Creeden, P. Ketteridge, Opt. Lett. 33, 315 (2008).

[10] J. Swiderski, M. Maciejewska, Appl. Phys. B 109, 177 (2012).

[11] J. Swiderski, M. Michalska, Laser Phys. Lett. 10, 035105 (2013).

[12] J. Swiderski, M. Michalska, G. Maze, Opt. Expr. 21, 7851 (2013). 